\title{
Corpus Callosum Subdivision Based on a Probabilistic Model of Inter-hemispheric Connectivity
}

\author{
Martin A. Styner ${ }^{1,2}$, Ipek Oguz ${ }^{1}$, Rachel Gimpel Smith ${ }^{2}$, \\ Carissa Cascio $^{2}$, and Matthieu Jomier ${ }^{1}$ \\ 1 Dept. of Computer Science, Univ. of North Carolina, Chapel Hill NC 27599, USA \\ ${ }^{2}$ Dept. of Psychiatry, Univ. of North Carolina, Chapel Hill NC 27599, USA
}

\begin{abstract}
Statistical shape analysis has become of increasing interest to the neuroimaging community due to its potential to locate morphological changes. In this paper, we present the a novel combination of shape analysis and Diffusion Tensor Image (DTI) Tractography to the computation of a probabilistic, model based corpus callosum (CC) subdivision. The probabilistic subdivision is based on the distances of arc-length parameterized corpus callosum contour points to trans-callosal DTI fibers associated with an automatic lobe subdivision. Our proposed subdivision method is automatic and reproducible. Its results are more stable than the Witelson subdivision scheme or other commonly applied schemes based on the CC bounding box. We present the application of our subdivision method to a small scale study of regional CC area growth in healthy subjects from age 2 to 4 years.
\end{abstract}

\section{Introduction}

Quantitative morphologic assessment of individual brain structures is often based on global volume and area measurements, which are intuitive features as they may explain atrophy or dilation due to illness. On the other hand, structural changes at specific locations are not sufficiently reflected in volume and area measurements. Shape analysis has thus become of increasing interest to the neuroimaging community. In this paper, shape analysis is employed to compute a probabilistic subdivision model of the Corpus Callosum(CC).

The corpus callosum is the major commisural pathway between the hemispheres and plays an integral role in relaying sensory, motor and cognitive information from homologous region in the two hemispheres. It has been a structure of much interest in neuroimaging studies of normal development [1, schizophrenia 2], autism, bipolar and unipolar disorder. In-vivo assessment of the commisural pathways through the $\mathrm{CC}$ is difficult, but can be approximated using Diffusion Tensor Imaging (DTI) and Tractography [345] (see Figure 1).

The computation of regional volumes and areas based on subdivision schemes of anatomical structures is quite common in neuroimaging. Most common subdivision protocols are executed manually by relabeling an already segmented 

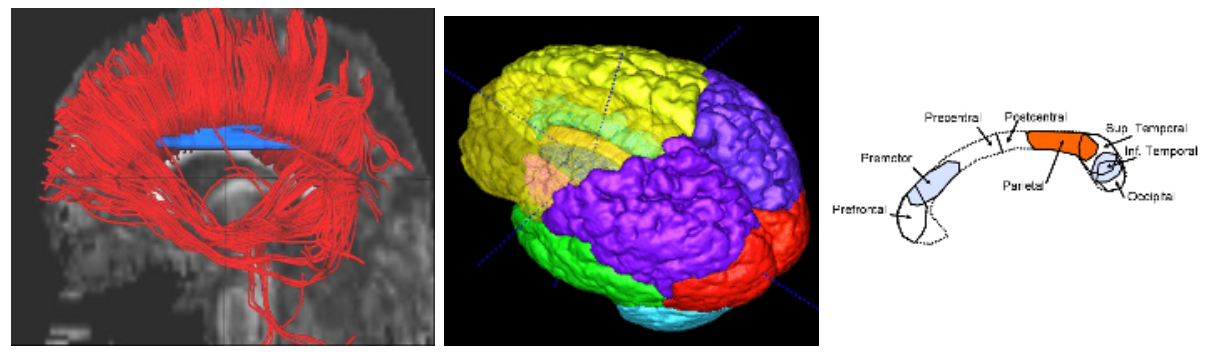

Fig. 1. Left: Visualization of interhemispheric, trans-callosal DTI Fibers. Middle: Result of an automatic lobe subdivision. Right: Schematic subdivision based on neurohistological studies 6].

structure into subregions. These methods are time-consuming, not reproducible and subjective. Further, they are often based on the structure's bounding box and are thus likely to mix different parts of the structure into the same subdivision due to the non-convex shape of most anatomical structures. Subdivision schemes can also be categorized into hard and probabilistic subdivisions. A hard subdivision assigns a single regional label for every image element. A probabilistic subdivision on the other hand assigns multiple labels with individual probabilistic weights for each element.

The currently most widely applied subdivision scheme for the $\mathrm{CC}$ was originally proposed by Witelson [6] and is motivated by neurohistological studies. It has been adapted in many studies [7]2. The Witelson based subdivisions use somewhat arbitrarily defined hard subdivision boundaries and is often still applied manually, even though automatic and probabilistic methods exist 8 . To our knowledge probabilistic subdivision methods in combination with fiber connectivity information have not been proposed before.

In this paper we propose a novel model based probabilistic subdivision scheme of the CC. The subdivision model described in section 2.1 is computed as the average model of a training population of automatic cortical lobe subdivisions propagated via inter-hemispheric, trans-callosal DTI fibers. The subdivision model is then applied to a small study of CC area growth in healthy children.

\section{Methods}

Subjects and Image Acquisition: There are 2 mutually exclusive sets of subjects used in this paper: one for the computation of the subdivision model and one for the small study on normal growth. The subdivision model was built from 5 different subjects of a larger database of healthy (2 cases), autistic (2) and developmentally delayed children (1) at age of 2 years (2) and 4 years (3). The growth study was computed on 3 additional healthy subjects with scans at age 2 and age 4. All subjects were scanned on the same GE 1.5 Tesla Sigma Advantage MR system. The structural MRI (sMRI) dataset was acquired using a 3D IR Prepped SPGR protocol with a $256 \times 256 \times 124$ image matrix at $0.9375 \times 0.9375 \times 1.5 \mathrm{~mm}$ res- 
olution. The DTI dataset was acquired using a 12 direction, 4 repetition DTI sequence with a $128 \times 128 \times 30$ image matrix at $1.875 \times 1.875 \times 4.2 \mathrm{~mm}$ resolution.

Corpus Callosum Segmentation: Our automatic segmentation of the CC from the sMRI data is an extension of Kelemen's 2D Fourier descriptor based Active Shape Model [9]. The shape model is described with complex Fourier descriptors up to degree 11. It was derived from a large, mixed population of adult controls, schizophrenics, pediatric controls and autistics. Based on a prior automatic tissue segmentation [10] the initial values for position, scale and grayscale normalization were computed automatically. From these initial values, the CC segmentation is performed in 2 steps: first within a larger search region $(6 \mathrm{~mm}$ along each profile) using a fully constrained model deformation, then secondly within a small search region (1 mm along each profile) using an unconstrained deformation. Each step is computed until convergence. We applied this method so far to over 150 pediatric cases with less than $2 \%$ cases that needed manual interaction in the segmentation process.

\section{Correspondence via Fourier Descriptors (arc-length parametrization):}

The segmentation procedure yields Fourier coefficients with an inherent correspondence based on its arc-length parametrization. The start-point for the arclength parametrization is given by the first order ellipse. The fourier descriptor were then uniformly sampled into a single polygon curve (100 points, spacing along curve is about $0.75 \mathrm{~mm}$ ).

Alignment and Scale: Alignment of the CC contours is achieved using the Procrustes [1] alignment method without scaling. We chose the iteratively computed average $\mathrm{CC}$ as the template of the Procrustes alignment. In the longitudinal study presented in this paper the CC contours were analyzed in their original scale and thus no scaling normalization was performed.

Model based subdivision: Our novel CC subdivision method is based on a prior subdivision model (described below in section 2.1). The subdivision model consists of 4 probabilistic maps that assign to each contour point $C(x)$ the probabilities $p_{i}(x)$ to belong to any of the 4 connectivity based subdivisions $S_{i}$. These probabilities are assigned to the contour points of each individual CC contour using the contour correspondence of the Fourier Descriptors. Our model subdivides thus not the full cross-section of the CC, but rather only its contour. The subdivision probabilities for the whole CC cross section are determined by closest point correspondence to the contour. This closest point operation results in the probabilistic area maps for the CC cross-section(see Figure 4). From the probabilistic area maps, the area values of the 4 regions are computed by simple summation. The computation of these probabilistic areas is automatic and reproducible.

\subsection{Subdivision Model}

The subdivision model was built from 5 pediatric cases combining fiber tract information from DTI data, and cortical lobe subdivision and shape information from T1w sMRI data. In summary we first compute for each case its lobe 
subdivision and the CC segmentation. Then the lobe subdivision is used for the computation of the interhemispheric lobar DTI fiber tracts. The next step computes a distance-weighted probabilistic subdivision of each case's CC contour from the location of all tracts. The resulting probabilistic subdivisions are averaged to produce the final CC subdivision model.

During several steps of the model computation, the results of the previous steps is transformed from DTI to sMRI coordinate space or vice-versa. This transformation was computed using a fully affine registration of the sMRI image to the $B 0$ DTI image based on normalized mutual information [12].

In the first step of the model computation, we employ a fluid registration 13 to propagate the lobe parcellation from a single template to all sMRI images. The lobe subdivisions were then controlled by experts. Only in few cases manual corrections are necessary. The result of the lobe subdivision is a set of separate left and right hemispheric lobes: frontal, parietal, occipital and temporal lobe. As a next step, the CC is segmented using the Fourier Descriptor Active Shape Model.

The lobe subdivision and the CC segmentation serve as automatic selection regions for the source (lobes) and target (CC) of the DTI fiber tract computation. This results in 4 sets of fibers that originate in each of the lobes, pass through the $\mathrm{CC}$ and end up in the corresponding lobe of the other hemisphere. The fibers from the occipital and temporal lobes are joined as their fiber tracts are highly overlapping. The limiting factor for a higher degree of lobar subdivision is the moderate resolution of the DTI datasets employed in this study. The fibers of the frontal lobe are further subdivided using an interactive 3D DTI tract clustering and manipulation tool called FiberViewer, which was developed at our lab. This fiber subdivision creates two fiber sets, one with fibers that are anteriorly oriented (anterior-frontal fibers) and one with fibers that are superiorly oriented (posterior-frontal fibers). A reconstruction of the 4 sets of fibers computed for a sample case is visualized in Figure 2. The fiber sets are quite overlapping and thus we chose to describe the model as a probabilistic subdivision, which is clearly better suited than a hard subdivision.
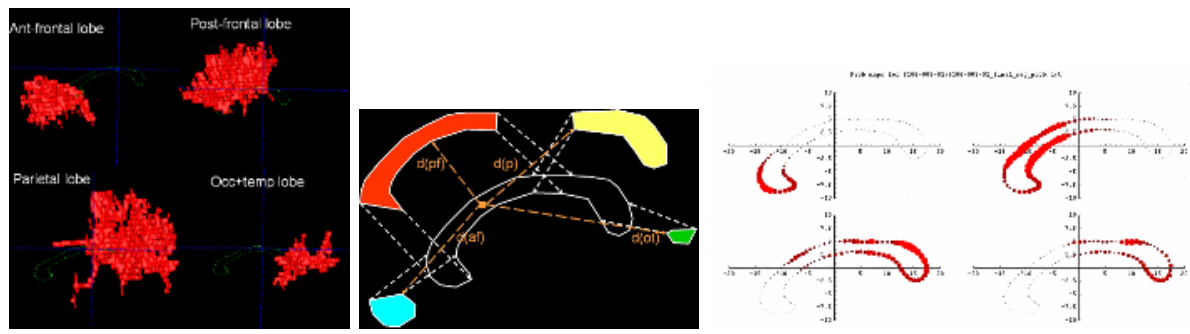

Fig. 2. Subdivision model computation. Left: Reconstruction of the fiber sets associated with each lobe in DTI coordinate space. Middle: Schematic visualization of the probability computation. Right: Sample CC contour probability map plotting disks of radii relative to the corresponding probability at each contour point. 
The 4 probabilistic subdivisions $p_{i}(x)$ of the CC contour are computed using the closest distances $d_{i}(x)=\operatorname{dist}(C(x), f(i))$ of every contour point $C(x)$ to the reconstructed 4 fiber sets $f(i): p_{i}(x)=\left(\operatorname{maxdist}-d_{i}^{2}(x)\right) / \sum_{i=0}^{4}\left(\operatorname{maxdist}-d_{i}^{2}(x)\right)$ where maxdist represents the maximal possible distance predetermined at the average length of the CC. The computation of the probabilities is schematically shown in Figure 2 along with the result of the probabilistic contour subdivision of a sample case. The contour subdivision is shown for each lobe separately plotting at each $\mathrm{CC}$ contour point disks with radii relative to the corresponding probability. The final probabilistic subdivision model is computed by linearly averaging the probabilities for each $\mathrm{CC}$ contour point across the training population.

\section{Results}

Probabilistic CC subdivision model: The contour probability maps of all 5 cases in the training population show a high similarity across all cases (see Figure [3A) and so does the final subdivision model(see Figure $3 \mathrm{C}$ ). The largest variabil-

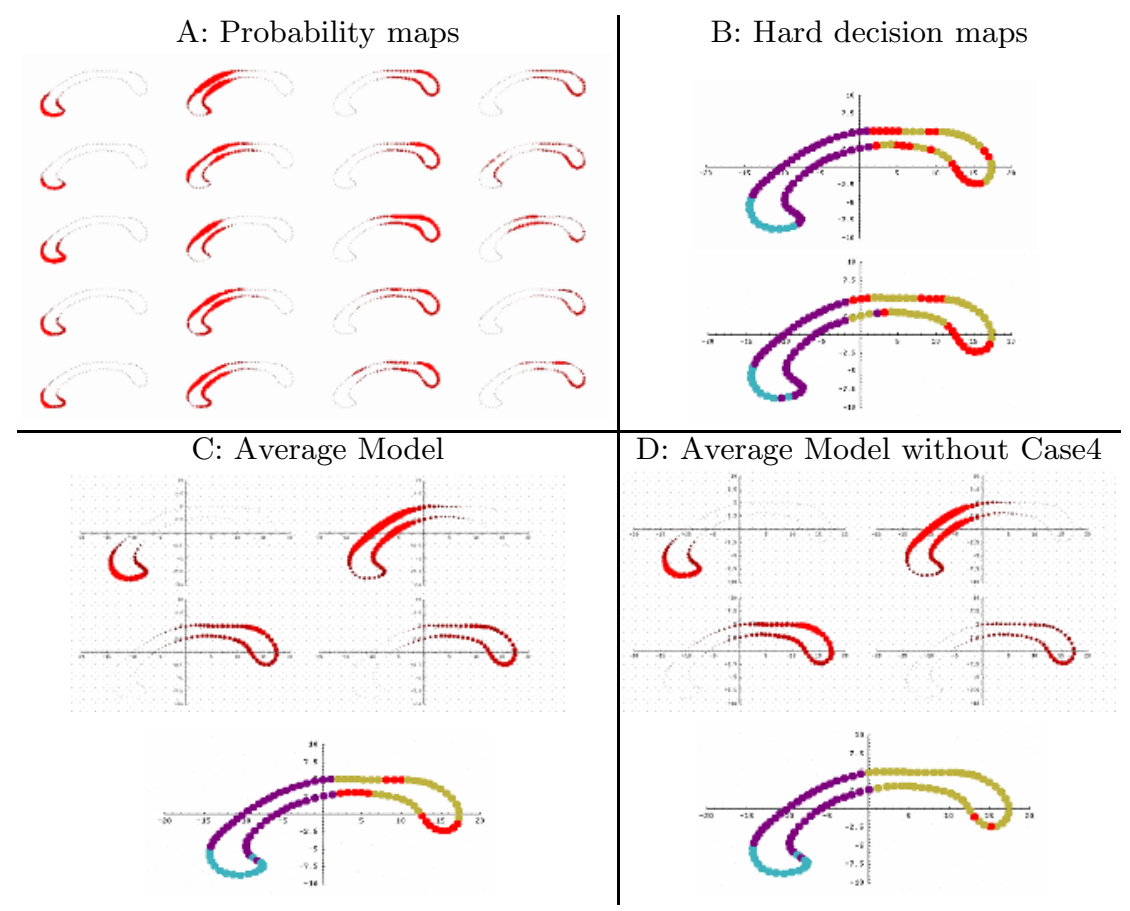

Fig. 3. Results of the subdivision model computation. A: Contour probability maps for all training cases. B: Hard decision maps for 2 selected training cases(cyan:anteriorfrontal, purple:posterior-frontal, brown:parietal, red:occipital-temporal lobe). C: Probabilistic and hard decision map of the final subdivision model. D: Probabilistic and hard decision map of the subdivision model excluding a single training case. 


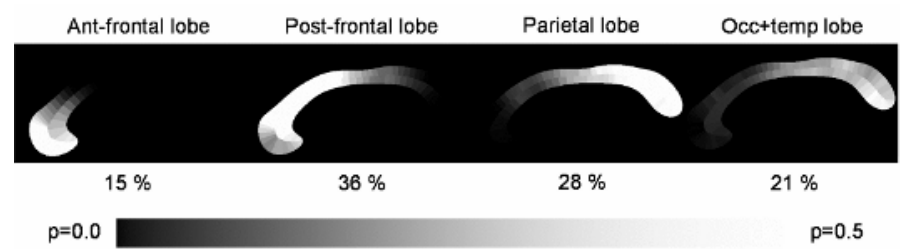

Fig. 4. Probabilistic area maps for a sample case. Each region is annotated with the respective probabilistic area percentage relative to the overall area.

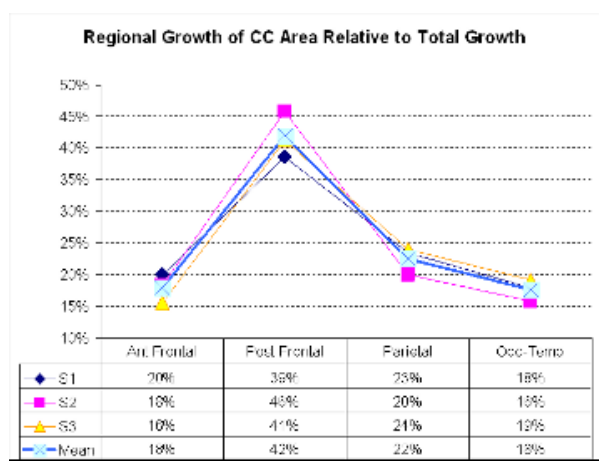

A

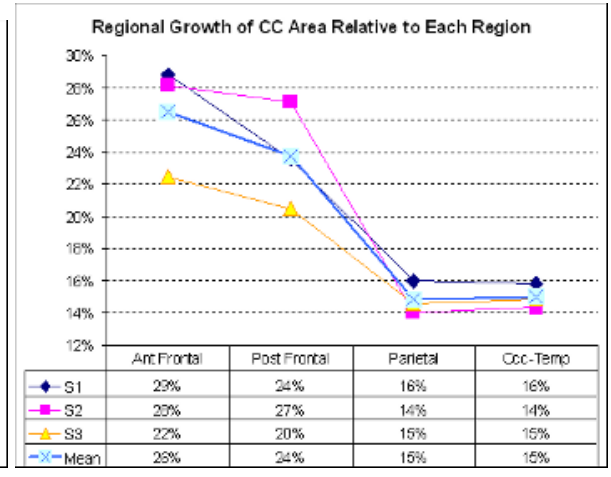

$\mathrm{B}$

Fig. 5. Relative growth curves of CC subdivision regions. Data from 3 healthy subjects along mean curves from age 2 to age 4 . A: Regional growth relative to the overall CC growth. B: Regional growth relative to the size of the corresponding region at age 2 .

ity seems to be present in the occipital-temporal lobe section. Alternatively to the probability maps, we also computed the hard decision maps by associating each contour point with a single region based on the highest probability. The high decision variability between the 2 selected cases shown in Figure $3 \mathrm{~B}$ clearly illustrates the main drawback of a hard decision map for the training cases. A similarly high variability is also present in a single leave-one-out experiment of a hard subdivision model computed from the final probability maps as shown in Figures $3 \mathrm{C}$ and D.

The result of the final subdivision model applied to a sample CC contour is shown in Figure 4. The occipital-temporal lobe region clearly shows a low probability in a relatively large region, which remains low even in the posteriormost sections of the CC. The resulting probabilistic area is relatively large $(21 \%$ for the shown sample case). In contrast, a hard decision model as illustrated in Figures $3 \mathrm{C}$ would compute a much lower area value and would thus highly underestimate the $\mathrm{CC}$ area associated with the occiptal-temporal lobe fibers.

Application of the model to a study of $\mathbf{C C}$ growth: In order to illustrate the potential of the subdivision model, we applied it to a small study of $\mathrm{CC}$ 
growth in 3 healthy children of age 2 to 4 . We applied the model after CC segmentation and computed the probabilistic area sum for the 4 regions. Figure 5] shows the resulting regional area growth $\mathrm{A}$ ) relative to the overall $\mathrm{CC}$ growth and $\mathrm{B}$ ) relative to the regional area of corresponding $\mathrm{CC}$ region at age 2 . The first shows the largest growth in the posterior-frontal lobe region and the smallest growth in the anterior-frontal lobe region. As the posterior-frontal region is overall the largest region and the anterior-frontal region is the smallest region, this growth curve plot can be misleading. The second plot captures the local growth more intuitively, as it shows the regional growth relative to the overall regional size. In this plot one can clearly see that the main growth is happening in the frontal lobe regions with the anterior-frontal lobe region experiencing the largest relative growth from age 2 to age 4 at $26 \%$.

\section{Discussion and Conclusion}

We present in this paper a novel method for the computation of a probabilistic subdivision model of an anatomical structure. The subdivision is not based on commonly applied arbitrarily subdivision boundaries based on the bounding box. Rather the subdivision is computed from probabilistic maps based on the distance to trans-callosal DTI fibers associated with a lobe subdivision.

Even though our CC subdivision model is based directly on DTI fiber connectivity information, the individual $\mathrm{CC}$ subdivisions are based on the geometric correspondence of the boundary to the subdivision model. On one hand, this scheme allows us to apply the subdivision model to retrospective $\mathrm{CC}$ data that lack an appropriate DTI dataset. On the other, if an appropriate DTI dataset is given, then we could directly compute the subdivision from the DTI fibers. For our present clinical studies either without DTI data or only with low-resolution DTI data the choice of a probabilistic subdivision model is the optimal method. In our future studies we are planning to recompute this subdivision model for a higher resolution data and investigate the direct computation of the CC subdivision from the DTI fibers.

The regions in our subdivision model are quite similar to those of the Witelson subdivision model [6], which is motivated by neurohistological studies. In contrast to the Witelson method is more stable due to the probabilistic nature of the subdivision and has been directly computed from connectivity information.

We are currently using the subdivision model to study regional CC growth in a large neurodevelopmental study of autistic, developmentally delayed and healthy subjects from age 2 to 4 . For a subset of this study additional relatively low-resolution DTI data is also available and we plan to use the model to study regional histograms of DTI properties such as Geodesic Anisotropy. We further plan to employ the model for the computation of regionally cumulative shape measurements, such as the mean distance between the mean contours of two populations in every CC subdivision region.

The results of the small scale study of callosal growth from year 2 to 4 is quite preliminary due to the low number of subjects. The high similarity of the results 
in all cases is though suggesting that the frontal lobe regions experience a larger growth than those of the posterior lobes in that stage of healthy development.

Acknowledgment. We are thankful to Sean Ho for the original corpus callosum segmentation software. We would further like to thank Daniel Rueckert for the affine registration tools and Sarang Joshi for the fluid registration program. This research is supported by the UNC Neurodevelopmental Disorders Research Center HD 03110.

\section{References}

1. Thompson, P., Giedd, J., Woods, R., MacDonald, D., Evans, A., Toga, A.: Growth patterns in the developing brain detected by using continuum mechanical tensor maps. Nature 6774 (2000) 190-3

2. Keshavan, M., Diwadkar, V., Harenski, K., Rosenberg, D., Sweeney, J., Pettegrew, J.: Abnormalities of the corpus callosum in first episode, treatment naive schizophrenia. J Neurol Neurosurg Psychiatry 6 (2002) 757-60

3. Fillard, P., Gilmore, J., Lin, W., Piven, J., Gerig, G.: Quantitative analysis of white matter fiber properties along geodesic paths. In: MICCAI. Number 2879 in Lecture Notes in Computer Science (2003) 16-23

4. Xu, D., Mori, S., Solaiyappan, M., van Zijl, P., Davatzikos, C.: A framework for callosal fiber distribution analysis. NeuroImage 17 (2002) 11311143

5. Gee, J., Zhang, H., Dubb, A., Avants, B., Yushkevich, P., Duda, J.: Anatomy-based visualizations of diffusion tensor images of brain white matter. In: Visualization and Image Processing of Tensor Fields. (2005)

6. Witelson, S.: Hand and sex differences in the isthmus and genu of the human corpus callosum. a postmortem morphological study. Brain 3 (1989) 799-835

7. Narr, K., Thompson, P., Sharma, T., Moussai, J., Cannestra, A., Toga, A.: Mapping morphology of the corpus callosum in schizophrenia. Cereb Cortex 1 (2000) 40-9

8. Bermudez P, Z.R.: Sexual dimorphism in the corpus callosum: methodological considerations in mri morphometry. Neuroimage 13 (2001) 1121-30

9. Székely, G., Kelemen, A., Brechbühler, C., Gerig, G.: Segmentation of 2-D and 3-D objects from MRI volume data using constrained elastic deformations of flexible Fourier contour and surface models. Medical Image Analysis 1 (1996) 19-34

10. Van Leemput, K., Maes, F., Vandermeulen, D., Suetens, P.: Automated modelbased tissue classication of $\mathrm{mr}$ images of the brain. IEEE Transactions on Medical Imaging 18 (1999) 897-908

11. Bookstein, F.: Shape and the Information in Medical Images: A Decade of the Morphometric Synthesis. Comp. Vision and Image Under. 66 (1997) 97-118

12. Rueckert, D., Frangi, A.F., Schnabel, J.A.: Automatic construction of 3d statistical deformation models of the brain using non-rigid registration. IEEE Transactions on Medical Imaging 8 (2003) 1014-1025

13. Joshi, S., Davis, B., Jomier, M., Gerig, G.: Unbiased diffeomorphic atlas construction for computational anatomy. NeuroImage 23 (2004) S151-S160 